Research Article

\title{
Serum lipid profile in non-polycystic ovary syndrome and polycystic ovary syndrome women: a comparative and correlational study
}

\author{
Nimish R. Halasawadekar ${ }^{1}$, Jaiprakash B. Ramanand ${ }^{2}$, Sunita J. Ramanand ${ }^{1}$, \\ Girish T. Raparti ${ }^{3}$, Praveenkumar T. Patil ${ }^{4}$, Ruchi D. Shah ${ }^{1}$, Arvind V. Kumbhar ${ }^{1}$
}

${ }^{1}$ Department of Pharmacology, GMC Miraj, Sangli,

Maharashtra, India,

${ }^{2}$ Department of Pharmacology, R.C.S.M. Government Medical College, Kolhapur, Maharashtra, India,

${ }^{3}$ Department of Pharmacology Ashwini Rural Medical College, SH51, Kumbhari, Solapur, Maharashtra, India, ${ }^{4}$ Department of Pharmacology, Shri Bhausaheb Hire Medical College, Dhule, Maharashtra, India

Received: 08 December 2015 Accepted: 07 January 2016

*Correspondence to: Dr. Nimish R Halasawadekar, Email: pharmac.gmc.miraj @gmail.com

Copyright: (C) the author(s), publisher and licensee Medip Academy. This is an openaccess article distributed under the terms of the Creative Commons Attribution NonCommercial License, which permits unrestricted noncommercial use, distribution, and reproduction in any medium, provided the original work is properly cited.

\begin{abstract}
Background: Polycystic ovary syndrome (PCOS), in addition to impaired ovulation, also affects metabolic pathways. Dyslipidemia, occurring in PCOS women leads to cardiovascular diseases in them. The purpose of the present study was to compare lipid profile and its correlation with biochemical and hormonal parameters in PCOS and non PCOS women, to analyse the correlation of lipid profile with hirsutism and body mass index (BMI) in PCOS women. Methods: The present study includes 68 women divided into non PCOS groups $(n=30)$ and PCOS $(n=38)$ as defined by Rotterdam criteria. PCOS group further divided into overweight / obese $(n=23)$ and normal weight subgroups $(n=15)$. Lipid profile, fasting blood glucose and hormonal profile were done in all the groups.

Results: TG and TC/HDL ratio were significantly high in PCOS group. Hirsute patients had raised LDL levels as compared to non-hirsute. LDL showed positive significant correlation with insulin, HOMA, testosterone in PCOS group. TC was significantly positively correlated with insulin and HOMA in PCOS group. In both normal weight and overweight / obese PCOS subgroups, LDL had positive and significant correlation with testosterone. TC showed positive and significant correlation with HOMA in overweight/obese subgroup. HDL showed positive and highly significant correlation with FSH in normal weight PCOS.

Conclusions: Hyperandrogenism in PCOS may be additionally marked by raised LDL. Overweight/obese PCOS subgroup may be prone to dyslipidemia as well as deranged glucose homeostasis, thereby making it an important therapeutic target.
\end{abstract}

Keywords: PCOS, Dyslipidemia, Correlation, Hirsuitism

\section{INTRODUCTION}

Polycystic ovary syndrome (PCOS) is one of the most common disorders in women of childbearing age. One to five percent of female population with 15 to 25 years suffers from PCOS and $10 \%$ women of reproductive age. ${ }^{1,2}$ In addition to impaired ovulation, it also affects metabolic pathways.
Obesity, insulin resistance (IR) and dyslipidemia, which may predispose patients to metabolic syndrome, are common in PCOS. ${ }^{3,4}$ Dyslipidemia is found in women with PCOS, independently of the excess weight. ${ }^{5}$ Women with PCOS have a greater prevalence of atherosclerosis and cardiovascular diseases and estimated seven fold increased risk for myocardial infarction. ${ }^{6-8}$ 
The purpose of the present study was to compare lipid profile and its correlation with biochemical and hormonal parameters in non PCOS and PCOS women. The study also analyses the correlation of lipid profile with hirsutism and body mass index (BMI) in PCOS women.

\section{METHODS}

The present study is an observational study. This study was conducted in 30 apparently healthy women (non PCOS group) and 38 newly diagnosed post pubertal PCOS women of reproductive age (PCOS group) in a tertiary care hospital. The women in non PCOS group were regularly menstruating and showed normal ultrasonography (USG). The diagnosis of PCOS was fulfilled as per Rotterdam criteria. Presence of at least two criteria from clinical, hormonal and abdominal USG category was considered diagnostic of PCOS. ${ }^{9}$

Adult Treatment Panel III (ATP III) considers levels of low density lipoproteins (LDL) $<100 \mathrm{mg} / \mathrm{dL}$ as optimal, $100-129 \mathrm{mg} / \mathrm{dL}$ as near optimal, triglyceride $(\mathrm{TG})<150$ $\mathrm{mg} / \mathrm{dL}$ as normal, total cholesterol (TC) $<200 \mathrm{mg} / \mathrm{dL}$ as desirable and high density lipoproteins (HDL) $\geq 50 \mathrm{mg} / \mathrm{dL}$ as normal for women. Dyslipidemia includes LDL levels as borderline high (130-150mg/dL), high (160-189 $\mathrm{mg} / \mathrm{dL})$ and very high $(\geq 190 \mathrm{mg} / \mathrm{dL}) ; \mathrm{TG}$ as borderline high (150-199 mg/dL), high (200-499 mg/dL) and very high (>500 mg/dL); TC as borderline high (200-239 $\mathrm{mg} / \mathrm{dL})$, high $(\geq 240 \mathrm{mg} / \mathrm{dL})$; HDL levels $(<50 \mathrm{mg} / \mathrm{dL})$ is defined as a marginal risk factor in women. ${ }^{10}$ TC:HDL $>4.4$ was also considered in dyslipidemia.

\section{Inclusion criteria}

Women with complain of irregular menses or oligomenorrhea (absence of menses for 35-182 days) or amenorrhea (absence of menses for $>182$ days), signs or symptoms of hyperandrogenism, abdominal USG showing at least 12 follicles (two to nine $\mathrm{mm}$ in diameter) arranged peripherally around a dense core of ovarian stroma or scattered throughout an increased amount of stroma were enrolled in the study. ${ }^{11}$

\section{Exclusion criteria}

Patients having any other major systemic illness including systemic inflammatory diseases, congenital adrenal hyperplasia, hyperprolactinemia, acromegaly, functional hypothalamic amenorrhea were excluded from the study.

The study was approved by the Institutional Ethics Committee. All the guidelines of Declaration of Helsinki were followed. Detailed menstrual history, marital status, parity were recorded in both the groups. The study subjects were clinically examined, subjected to pelvic ultrasonography and investigated for the endocrine and metabolic laboratory parameters. ${ }^{11}$
Anthropometric measurements were taken. Height and weight were measured. Body mass index (BMI) was calculated as weighing kilograms divided by the square of height in meters $\left(\mathrm{kg} / \mathrm{m}^{2}\right) .{ }^{11}$ Cut-off of BMI as Standard Consensus Statement for Indian population was considered, i.e. Normal weight (NW) BMI: 18.0-22.9 $\mathrm{kg} / \mathrm{m}^{2}$; Overweight (OW): 23.0-24.9 kg/m²; Obesity(OB): $>25 \mathrm{~kg} / \mathrm{m}^{2}{ }^{12}$ PCOS group, based on BMI, was stratified into normal weight (NWT) and overweight/obese (OW/OB) PCOS subgroups.

\section{Assay methods}

Lipid profile was estimated by quantitative enzymatic colorimetric method (GPO-PAP methodology) using Agappe diagnostic kits. This included low-densitylipoprotein (LDL), serum triglyceride (TG), total cholesterol (TC), high-density-lipoprotein (HDL) and TC/HDL ratio.

Serum gonadotropins levels were measured by sandwich immunoassay method. This included luteinizing hormone (LH), follicle stimulating hormone (FSH), LH: FSH ratio and testosterone. Serum insulin was determined by in vitro quantitative determination of hormones was carried by electrochemiluminescence immunoassay method (Roche-Hitachi Cobas e 411). Blood glucose was measured on autoanalyser (Vital Scientific Microlab 300) using oxidase method. Samples for fasting blood glucose (FBG) and fasting serum insulin levels were taken after overnight fasting. Homeostatic model assessment (HOMA) was calculated as marker of IR. HOMA value $>1.9$ was considered as presence of insulin resistance.

In the non PCOS group, the investigations were done from $6^{\text {th }}$ to $12^{\text {th }}$ day of menstrual cycle. In PCOS group, the investigations carried out were independent of day of menses because of irregular menses.

\section{Statistical tests}

Data was analyzed using statistical software (Graph pad prism version 5). Intergroup data was compared by unpaired ' $t$ ' test. Spearman test was used for Correlation. 'p' value less than 0.05 was considered significant.

\section{RESULTS}

Mean age of non PCOS group and PCOS group were $20.73 \pm 2.100$ and $22.29 \pm 3.683$ years respectively.

Mean TC/HDL ratio was significantly higher in PCOS group (4.335 \pm 0.64$)$ as compared to the non PCOS group $(3.976 \pm 0.55)(\mathrm{p}$ value $=0.0172 *)$.

In the present study, LDL, TG, TC and HDL levels were comparable in non PCOS and PCOS groups. 
Table 1 : Comparison of age and lipid profile both in non PCOS and PCOS women.

\begin{tabular}{|ll|lllll|}
\hline & AGE & LDL & TG & TC & HDL & TC/HDL \\
\hline Non-PCOS (Mean \pm SD.) & $20.73 \pm 2.100$ & $89.70 \pm 15.61$ & $109.0 \pm 16.35$ & $160.3 \pm 13.53$ & $41.93 \pm 8.35$ & $3.97 \pm 0.55$ \\
\hline PCOS (Mean \pm SD.) & $22.29 \pm 3.683$ & $90 \pm 21$ & $124 \pm 31$ & $167 \pm 21$ & $39 \pm 4.7$ & $4.3 \pm 0.64$ \\
\hline p value & $0.0431^{*}$ & 0.954 & $0.021^{*}$ & 0.117 & 0.0714 & $0.017^{*}$ \\
\hline
\end{tabular}

*significant

In PCOS group, patients with hirsutism showed higher mean lipid profile as compared to patients without hirsutism except for HDL $(38.79 \pm 4.775$ in patient with hirsutism versus $39.13 \pm 4.721$ in patient without hirsutism).

In PCOS group, lipid profile in overweight/ obese subgroup showed more mean value as compared to normal weight subgroup.

Table 2: Comparison between lipid profile in PCOS group based on hirsutism and body mass index.

\begin{tabular}{|c|c|c|c|c|}
\hline & \multicolumn{2}{|c|}{ Hirsutism } & \multicolumn{2}{|l|}{ BMI } \\
\hline & $\begin{array}{l}\text { Absent } \\
n=24\end{array}$ & $\begin{array}{l}\text { Present } \\
n=14\end{array}$ & $\begin{array}{l}\mathrm{NW} \\
\mathrm{n}=15\end{array}$ & $\begin{array}{l}\mathrm{OW} / \mathrm{OB} \\
\mathrm{n}=23\end{array}$ \\
\hline LDL & $\begin{array}{l}85.50 \pm \\
14.15\end{array}$ & $\begin{array}{l}98.36 \pm \\
27.56\end{array}$ & $\begin{array}{l}86.40 \pm \\
11.95\end{array}$ & $\begin{array}{l}92.74 \pm \\
24.85\end{array}$ \\
\hline $\mathrm{p}$ value & 0.0646 & & 0.3647 & \\
\hline $\mathrm{TC}$ & $\begin{array}{l}163.2 \pm \\
20.48\end{array}$ & $\begin{array}{l}174.6 \pm \\
21.97\end{array}$ & $\begin{array}{l}163.0 \pm \\
17.59\end{array}$ & $\begin{array}{l}170.3 \pm \\
23.60\end{array}$ \\
\hline $\mathrm{p}$ value & 0.1169 & & 0.3149 & \\
\hline TG & $\begin{array}{l}119.5 \pm \\
30.21\end{array}$ & $\begin{array}{l}131.1 \pm \\
31.57\end{array}$ & $\begin{array}{l}119.9 \pm \\
29.47\end{array}$ & $\begin{array}{l}126.3 \pm \\
32.08\end{array}$ \\
\hline $\mathrm{p}$ value & 0.2653 & & 0.5435 & \\
\hline HDL & $\begin{array}{l}39.13 \pm \\
4.721\end{array}$ & $\begin{array}{l}38.79 \pm \\
4.775\end{array}$ & $\begin{array}{l}38.87 \pm \\
4.549\end{array}$ & $\begin{array}{l}39.09 \pm \\
4.861\end{array}$ \\
\hline $\mathrm{p}$ value & 0.8327 & & 0.8895 & \\
\hline TC/HDL & $\begin{array}{l}4.215 \pm \\
0.6255\end{array}$ & $\begin{array}{l}4.542 \pm \\
0.6271\end{array}$ & $\begin{array}{l}4.232 \pm \\
0.5553\end{array}$ & $\begin{array}{l}4.403 \pm \\
0.6902\end{array}$ \\
\hline $\mathrm{p}$ value & 0.1285 & & 0.4279 & \\
\hline
\end{tabular}

$\mathrm{n}=38$ in PCOS group.

In non PCOS group, LDL showed positive correlation with LH, FSH, LH/FSH ratio and testosterone and negative correlation with FBG, Insulin and HOMA.

In PCOS group, LDL showed inverse significant correlation with endocrine and metabolic parameters except testosterone which shows significant positive correlation $(\mathrm{r}=0.52)$. There is no correlation noted between LDL and LH levels in cases $(r=0)$.

In non PCOS group, TG is positively correlated with all endocrine and metabolic parameters.

In PCOS group, TG showed positive correlation Insulin $(\mathrm{r}=0.248)$ and $\operatorname{HOMA}(\mathrm{r}=0.152)$.

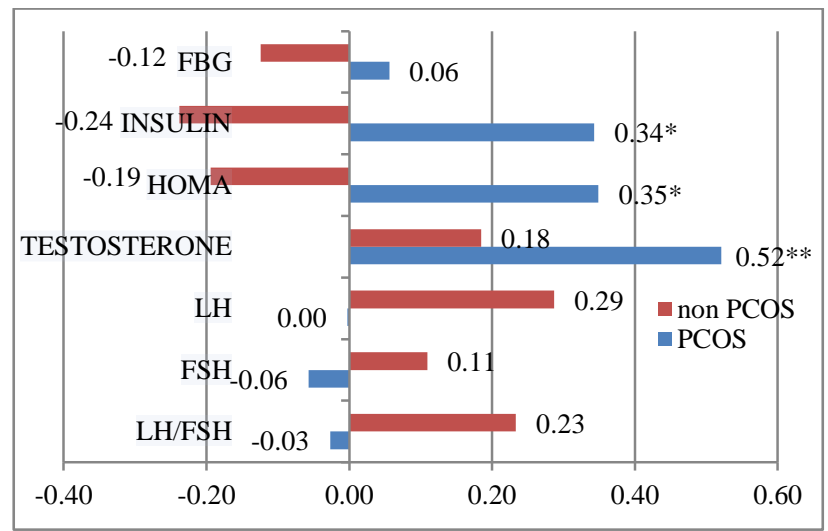

*Significant; Correlation coefficient ' $r$ ' values are mentioned. $n$ $=30$ in non PCOS group and $\mathrm{n}=38$ in PCOS group.

Figure 1: Correlation between LDL and endocrine, metabolic profile both in non PCOS and PCOS groups.

In non PCOS group, TC showed positive correlation with LH, FSH, LH/FSH, HOMA and Insulin while negative correlation with testosterone and FBG. In PCOS group, TC showed inverse correlation with $\mathrm{LH} / \mathrm{FSH}$ and testosterone.

In non PCOS group, HDL showed negative correlation with all endocrine and metabolic parameters except with FSH. In PCOS group, HDL showed inverse correlation with $\mathrm{LH}$ and FBG.

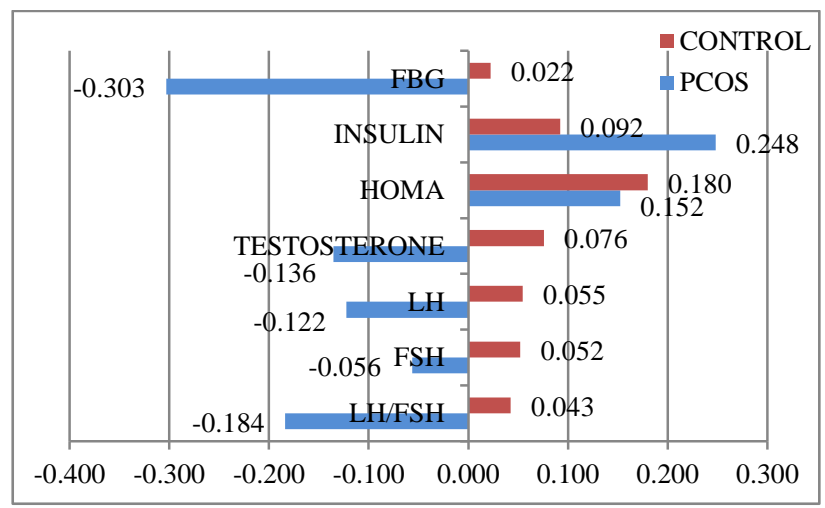

Correlation coefficient ' $\mathrm{r}$ ' values are mentioned; $\mathrm{n}=30$ in nonPCOS group and $n=38$ in PCOS group.

Figure 2: Correlation between triglyceride (TG) and endocrine, metabolic profile both in non PCOS and PCOS groups. 


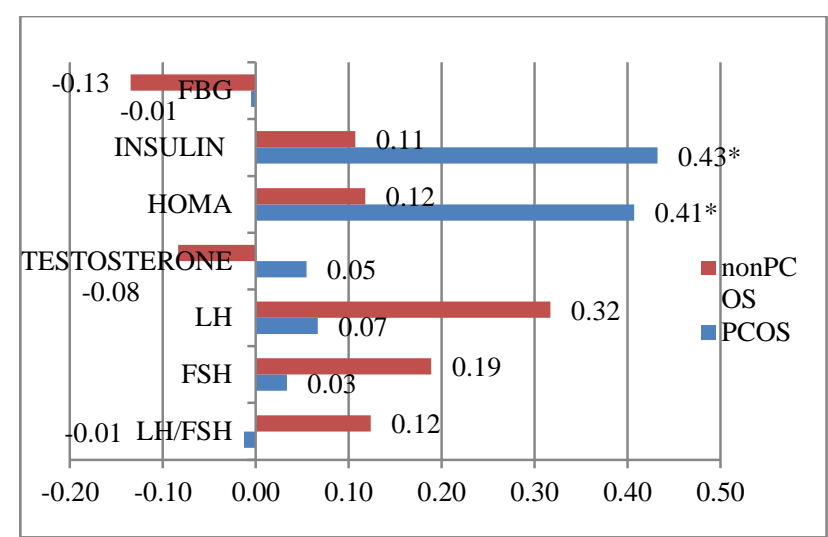

*Significant; Correlation coefficient ' $r$ ' values are mentioned; $\mathrm{n}$ $=30$ in non PCOS group and $n=38$ in PCOS group.

Figure 3: Correlation between total cholesterol and endocrine, metabolic profile both in nonPCOS and PCOS groups.

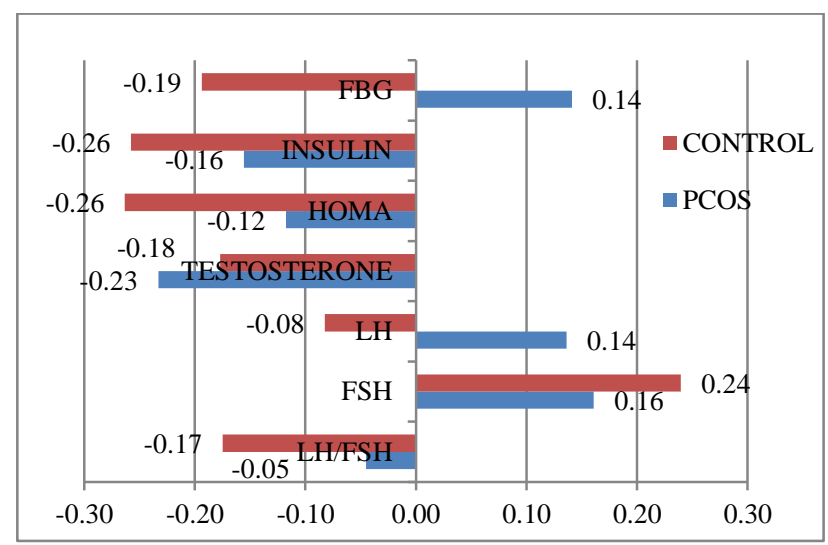

*Significant; Correlation coefficient ' $r$ ' values are mentioned. $\mathrm{n}$ $=30$ in nonPCOS group and $n=38$ in PCOS group.

Figure 4: Correlation between HDL and endocrine, metabolic profile both in non PCOS and PCOS groups.

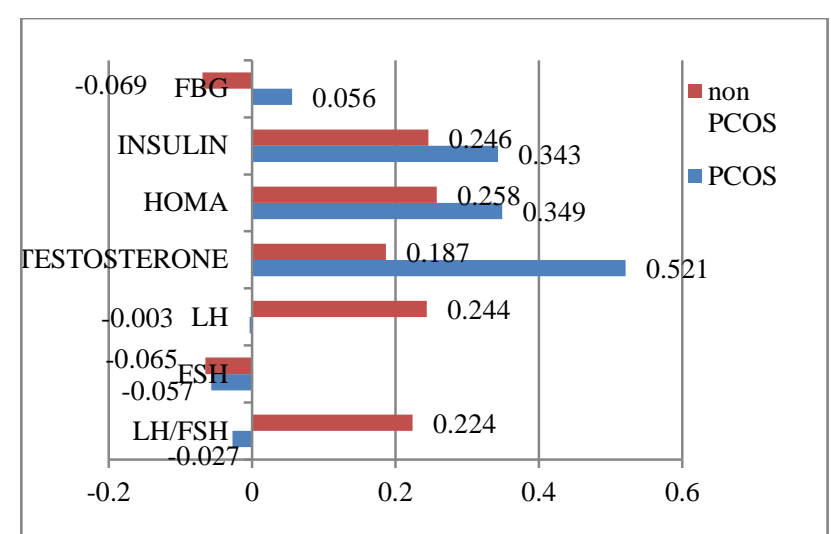

Correlation coefficient ' $\mathrm{r}$ ' values are mentioned; $\mathrm{n}=30$ in nonPCOS group and $n=38$ in PCOS group.

Figure 5: Correlation between TC/HDL and endocrine, metabolic profile both in non PCOS and PCOS groups.
In PCOS group, TC:HDL ratio showed inverse correlation with FBG and LH/FSH as compared to non PCOS group.

It has been found in NWT PCOS subgroup that LDL is positively correlated with endocrine and metabolic parameters except FSH where is negatively correlated $(\mathrm{r}=$ $-0.08)$.

In the OW/OB PCOS subgroup, LDL showed reverse correlation with $\mathrm{LH}$ and $\mathrm{LH} / \mathrm{FSH}$.

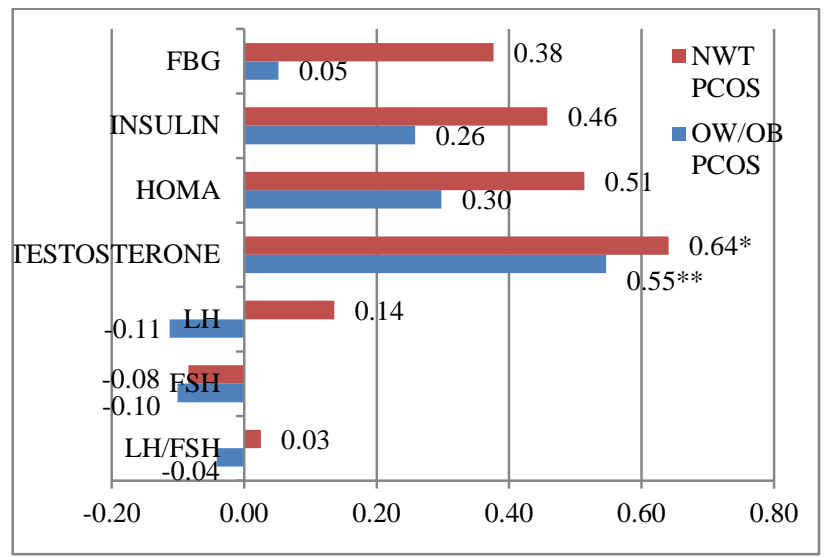

* Significant; Correlation coefficient ' $r$ ' values are mentioned; $n$ $=15$ in NWT PCOS group and $n=23$ in OW/OB PCOS group.

Figure 6: Correlation between LDL and endocrine, metabolic profile both in normal weight (NWT) PCOS and overweight/ obese (OW/OB) PCOS subgroups.

In NWT PCOS subgroup, TG showed negative correlation with all endocrine and metabolic parameters except $\mathrm{LH}$, FSH and Insulin. In OW/OB PCOS subgroup, TG showed inverse correlation with HOMA, LH and FSH.

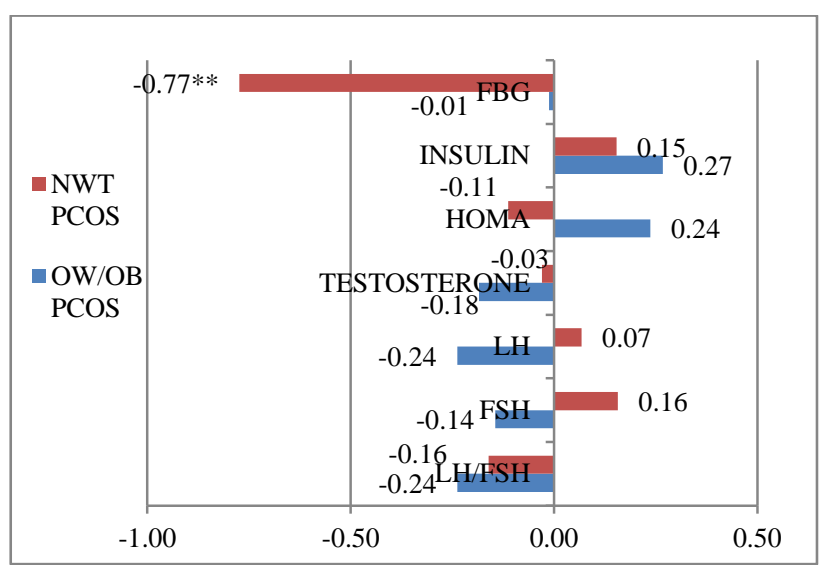

* Significant; Correlation coefficient ' $r$ ' values are mentioned. $\mathrm{n}$ $=15$ in NWT PCOS group and $n=23$ in OW/OB PCOS group.

Figure 7: Correlation between triglyceride (TG) and endocrine, metabolic profile both in normal weight (NWT) PCOS and overweight/obese PCOS (OW/OB) subgroups. 
In NWT PCOS subgroup, TC is positively correlated with all endocrine and metabolic profile parameters except LH/FSH, Similarly, in OW/OB PCOS subgroup, TC is positively correlated with all parameters except FSH.

There is an inverse correlation of TC with all endocrine and metabolic parameters in both NWT PCOS and OW/OB PCOS subgroups except FSH and LH/FSH ratio.

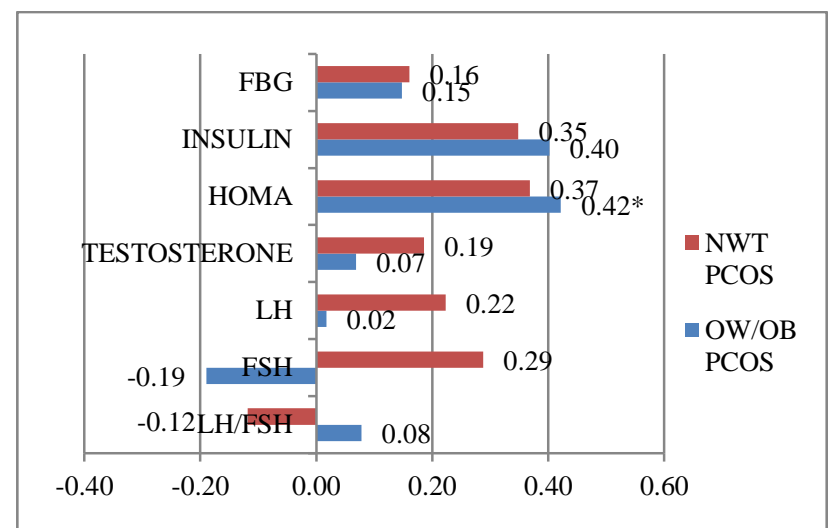

*Significant; Correlation coefficient ' $r$ ' values are mentioned; $n$ $=15$ in NWT PCOS group and $n=23$ in OW/OB PCOS group.

Figure 8: Correlation between Total cholesterol (TC) and endocrine, metabolic profile both in normal weight (NWT) PCOS and overweight/obese PCOS (OW/OB) subgroups.

In NWT PCOS subgroup, HDL showed positive correlation with FSH (r value-0.7**), LH and FBG. In OW/OB PCOS subgroup, HDL showed inverse correlation with FSH, HOMA and FBG.

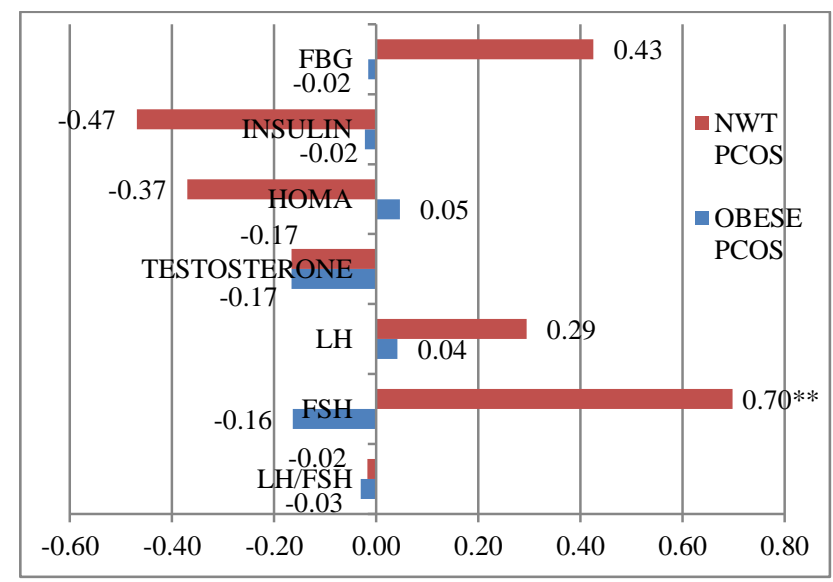

**Significant; Correlation coefficient ' $r$ ' values are mentioned; $\mathrm{n}=15$ in NWT PCOS group and $\mathrm{n}=23$ in OW/OB PCOS group.

Figure 9: Correlation between HDL and endocrine, metabolic profile both in normal weight (NWT) PCOS and overweight/obese (OW/OB) PCOS subgroups.

In OW/OB PCOS subgroup, TC/HDL ratio showed inverse correlation with $\mathrm{FBG}, \mathrm{FSH}$ and $\mathrm{LH} / \mathrm{FSH}$ ratio as compared to NW PCOS subgroup.

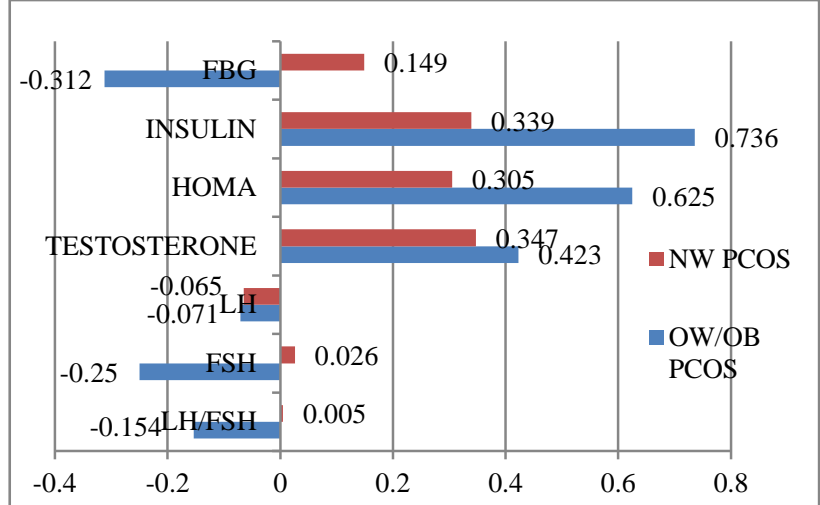

Correlation coefficient ' $r$ ' values are mentioned; $n=15$ in NWT PCOS group and $n=23$ in OW/OB PCOS group.

Figure 10: Correlation between TC/HDL and endocrine, metabolic profile both in normal weight (NWT) PCOS and overweight obese (OW/OB) PCOS subgroups.

\section{DISCUSSION}

Dyslipidemia is a common metabolic derangement in PCOS. The purpose of the present work was to compare lipid profile in PCOS and non PCOS Indian women as well as to study correlation of different serum lipoproteins with clinical presentation of PCOS and hormonal as well as glycemic biochemical parameters.

\section{Baseline data}

In the present study, the mean serum levels of LDL, TG, and TC were higher in PCOS group as compared to non PCOS group. HDL levels were less than normal values in both non PCOS and PCOS groups. The reduction in HDL levels PCOS group was to a greater extent. These findings were in accordance with Annamaria et al. ${ }^{13}$ Studies of Nashwa et al and Jayshree et al also showed similar findings but were significant. ${ }^{14,15}$ According to Rajkhowa et al, the ratio of total cholesterol to HDL, a widely used index of cardiovascular risk, was significantly greater in PCOS than in non PCOS group. ${ }^{16}$ This finding was consistent with present study $\left(\mathrm{p}=0.0172^{*}\right)($ Table 1$)$

Present study shows that the occurrence of deranged serum lipids in PCOS women is mainly in the form of decreased HDL. All the PCOS women $(100 \%)$ had lower serum HDL levels. The serum TG levels of $28.9 \%$ PCOS women were raised. The occurrence of raised TC: HDL, TC and LDL were $26.31 \%, 0.07 \%$ and $0.052 \%$ respectively. The array of deranged serum lipid profile is similar to that reported in the previous study by Rocha MP et al. In their study, the most frequent abnormalities were low high-density lipoprotein cholesterol (HDL-C; $57.6 \%)$ and high triglyceride (TG) (28.3\%). HDL-C was significantly lower in all subgroups of women with PCOS when compared to the subgroups of normal women. ${ }^{17}$ The dyslipidemia in PCOS to some extent is attributed to the 
presence of insulin resistance in PCOS women. Insulin resistance and hyperinsulinemia affects serum lipid profile adversely by reducing HDL and increasing TG levels. ${ }^{18}$ The lesser mean serum HDL in PCOS women as compared to the non PCOS women failed to reach the level of significance, may be because some (26\%) women in the later group also showed reduced serum HDL levels (Table 1). The present results suggest that young Indian women are at the risk of deranged serum HDL and PCOS is a high risk group for the same. Life style modification including dietary habits to increase HDL should be advocated in young Indian women, more than ever in PCOS women.

Comparison of lipid profile in PCOS women based on BMI shows that the mean serum levels of LDL, TG, TC and TC: HDL are higher in overweight/obese PCOS women. Obesity accentuates lipid derangement in PCOS. Hirsute PCOS women showed higher mean serum LDL (near significant), TG, TC and TC: HDL (Table 2). Alexander Carolyn quoted that hyperinsulinemia and hyperandrogenemia cause adipocytes to undergo increased catecholamine-induced lipolysis and release of free fatty acids into the circulation. Increased free fatty acids in the liver stimulate secretion of very low-density lipoprotein (VLDL), which ultimately leads to hypertriglyceridemia. ${ }^{19}$ It is clear that obesity, insulin resistance and hyperandrogenism coexist in PCOS, and have independent and interactive effects on dyslipidemia, although the mechanisms of these interactions remain elusive. $^{20}$

In contrast to the non-PCOS women, PCOS women showed significant positive correlation between serum LDL and the glycemic parameters. The positive correlation between serum LDL and testosterone is significant in PCOS women (Figure 1). This in addition to the greater mean serum LDL levels in the hirsuite PCOS women as mentioned above endorses important role of testosterone in LDL metabolism in PCOS. The positive correlation between serum $\mathrm{TG}$ and insulin is greater in PCOS women as compared to the non PCOS women (Figure 2). The positive correlation between TC and serum insulin as well as HOMA is significant in PCOS women (Figure 3). Insulin affects lipid metabolism. The extent of correlation between serum lipids (LDL, TG, TC) and insulin as well as the indicator of insulin resistance HOMA is greater in PCOS women as compared to the non PCOS women. This suggest central role of insulin resistance in the derangement of these lipoproteins. As compared to non-PCOS women the degree of negative correlation between serum HDL and insulin, HOMA is lesser and that with testosterone is greater (Figure 4).

The correlational analysis in normal weight and overweight/obese PCOS women was compared. Both normal weight and overweight/obese PCOS women showed positive correlation between serum LDL and insulin, HOMA. The positive correlation between serum
LDL and testosterone was significant in both the subgroups of PCOS (Figure 6). The positive correlation between TC and HOMA was significant in overweight/obese women (Figure 8). Normal weight PCOS subgroup showed a highly significant positive correlation between serum HDL and FSH (Figure 9). The correlation between gonadotropins and lipid profile is not well known. In PCOS women, FSH levels are reduced. Reduced serum HDL and its positive correlation with FSH in normal weight PCOS women unwraps a new consideration in the gonadal milieu and lipid metabolism especially with respect to HDL. The present results show that to some extent normal weight PCOS and overweight PCOS women differ in lipoprotein metabolism and their correlation with hormonal parameters.

\section{CONCLUSION}

Dyslipidemia is a common metabolic disorder in PCOS women. It is mostly associated with obesity. Dyslipidemia is a major contributing factor for cardiovascular complications in PCOS women. In the present study, LDL may be an additional risk factor for hyperandrogenism in PCOS women because of its positive correlation with testosterone. The decreased level of HDL is seen in all PCOS women making it an important therapeutic target. The raised TC affects glucose homeostasis and lower levels of HDL affects ovulation to some extent.

Funding: The study was funded by medical research council of Maharashtra/Directorate medical education and research, Mumbai, under star research scheme Conflict of interest: None declared

Ethical approval: The study was approved by the Institutional Ethics Committee

\section{REFERENCES}

1. Padubidre VG, Daftary SN. Disorders of Ovary. Shaw's Text book of Gynaecology, $13^{\text {th }}$ edition, Elsevier publishers. 2004:353-4.

2. Franks S. Polycystic ovary syndrome. New England J Medicine. 1995;333:853-61.

3. Dunaif A, Segal KR, Futterweit W, Dobrjansky A. Profound peripheral insulin resistance, independent of obesity, in polycystic ovary syndrome. Diabetes. 1989;38(9):1165-74.

4. Kalra A, Nair S, Rai L. Association of obesity and insulin resistance with dyslipidemia in Indian women with polycystic ovarian syndrome. Indian J Med Sci. 2006;60(11):447-53.

5. Al-hakeim HK, Shaba'a SM, Kadheim MA. Is lipid profile in women with polycystic ovary syndrome related to calcium or magnesium in serum. Journal Kerbala University. 2009;7:150-7.

6. Guzick DS, Talbott EO, Sutton-Tyrrell K, Herzog HC, Kuller LH, Wolfson SK Jr. Carotid atherosclerosis in women with polycystic ovary 
syndrome: initial results from a case-control study. Am J Obstet Gynecol. 1996;174(4):1224-9.

7. Birdsall MA, Farquhar CM, White HD. Association between polycystic ovaries and extent of coronary artery diseases in women having cardiac catheterization. Ann Intern Med. 1997;126:32-5.

8. Dahlgren E1, Janson PO, Johansson S, Lapidus L, Oden A. Ovary syndrome and risk for myocardial infarction. Evaluated from a risk factor model based on a prospective population study. Acta Obstet Gynecol Scand. 1992;71(8):559-604.

9. Rotterdam group. Revised 2003 consensus on diagnostic criteria and long-term health risks related to polycystic ovary syndrome. Hum Reprod. 2004;19(1):41-7.

10. Third Report of the National Cholesterol Education Program (NCEP) Expert Panel on Detection, Evaluation, and Treatment of High Blood Cholesterol in Adults (Adult Treatment Panel III) final report. Circulation. 2002;106:3143-421.

11. Ramanad SJ, Ramanad JB, Jain SS, Raparti GT, Ghanghas RR, Halasawadekar NR, et al. Leptin in non PCOS and PCOS women: a comparative study. Int J Basic Clin Pharmacol. 2014;3(1):186-93.

12. Misra A, Chowbey P, Makkar BM, Vikram NK, Wasir JS, Chadha D, et al. Consensus Statement for Diagnosis of Obesity, Abdominal Obesity and the Metabolic Syndrome for Asian Indians and Recommendations for Physical Activity, Medical and Surgical Management. JAPI. 2009;57:163-70.

13. Fulghesu A, Magnini R (2012). Obesity Related Lipid Profile and Altered Insulin Incretion in Adolescent with Policystic Ovary Syndrome,
Dyslipidemia - From Prevention to Treatment, Prof. Roya Kelishadi (Ed.), ISBN: 978-953-307-904-2.

14. Abulhassan N, Dawlatly B, Hesham A, Abul Ela A, Abdel Kader N. Dyslipidemia and Insulin Resistance in Obese and Non-Obese Polycystic Ovarian Syndrome Women. Kasr el aini medical journal. 2007;13(1):107-16.

15. Jayasree R, Vijayalakshmi M. Altered lipid profile associated with polycystic ovarian syndrome women of Tamilnadu. The Bioscan. 2010;5(1):131-3.

16. Rajkhowa M, Neary RH, Kumpatla P, Game FL, Jones PW, Obhrai MS et al. Altered Composition of High Density Lipoproteins in Women with the Polycystic Ovary Syndrome. The Journal of Clinical Endocrinology \& Metabolism. 1997;82(10):3389-94.

17. Rocha MP, Marcondes JA, Barcellos CR, Hayashida SA, Curi DD, da Fonseca AM, et al. Dyslipidemia in women with polycystic ovary syndrome: incidence, pattern and predictors. Gynecological Endocrinology. 2011;27(10):814-9.

18. Goldberg IRA. Clinical review Diabetic Dyslipidemia: Causes and Consequences. JCE \& M. 2001;86:965-71.

19. Alexander CJ, Tangchitnob EP, Lepor NE. Polycystic ovary syndrome: a major unrecognized cardiovascular risk factor in women. Reviews in Obstetrics and Gynecology. 2009;2(4):232-9.

20. Diamanti-Kandarakis E, Papavassiliou AG, Kandarakis SA, Chrousos GP. Pathophysiology and types of dyslipidemia in PCOS. Trends in Endocrinology \& Metabolism. 2007;18(7):280-5.

Cite this article as: Halasawadekar NR, Ramanand JB, Ramanand SJ, Raparti GT, Patil PT, Shah RD et al. Serum lipid profile in non-polycystic ovary syndrome and polycystic ovary syndrome women: a comparative and correlational study. Int J Basic Clin Pharmacol 2016;5:105-11. 